International Mathematical Forum, 2, 2007, no. 11, 533 - 542

\title{
Invariant Functions for Discrete Derivatives and Their Applications to Solve Non-Homogenous Linear and Non-Linear Difference Equations ${ }^{1}$
}

\author{
N. Aliev, N. Azizi and M. Jahanshahi ${ }^{2}$ \\ Dept. of Math. - Islamic Azad University of Karadj Branch \\ Rajaee Shahr, Karadj, Tehran, Iran
}

\begin{abstract}
Linear and Non-linear difference equations are appeared in many fields of applied mathematics, engineering and physical problems such as natural phenomena, social and economical systems which have essentially discrete elements.

In this paper by making use of invariant functions for discrete derivatives [6], we present an analytical method to solve the general form of the $n$-th order non-homogenous linear difference equations and some non-linear difference equations.
\end{abstract}

\section{Mathematics Subject Classification: 34}

Keywords: Discrete derivatives, Invariant functions, Difference equations

\section{Introduction}

Unlike differential equations, difference equations theory neither in principles and methods of solving nor in theoretical discussions on existence and uniqueness of solution, hasn't been improved.

These equations which are discrete corresponded to differential equations, could be seen in subjects like combinatorics, graph theory and in general discrete systems.

\footnotetext{
${ }^{1}$ This research work has been supported by a grant from Islamic Azad University of Karaj branch.

${ }^{2}$ Corresponding author, e-mail: jahan_m15@yahoo.com
} 
In this paper we regard concept of discrete additive derivative to solve linear difference equations in form of analytic and by using discrete multiplicative derivative, nonlinear equations would be solved.

In addition to mathematics, these kinds of equations can be seen in mathematical models of physical, engineering, natural phenomena and social and economical systems which have essentially discrete elements.

If we could correspond discrete mathematical model to physical and natural problems, there would be natural conformity. Unfortunately as it was mentioned, there is no succeed in discrete methods and there are many limitations and troubles in expanding of solution methods.

\section{Linear difference equations}

To solve linear difference equations, we use the meaning of discrete additive derivative of discrete function

$$
f: A \subseteq Z \rightarrow R \quad x \in Z
$$

by

$$
f^{\prime}(x)=f(x+1)-f(x)
$$

and its invariant function for this kind of derivative are as follows; $f(x)=2^{x}$. [5], [6]

Now consider the differential equations that their derivatives are discrete additive:

$$
a_{n} y^{(n)}(x)+a_{n-1} y^{(n-1)}(x)+\ldots+a_{1} y^{\prime}(x)+a_{0} y(x)=0 \quad x \in Z \quad x \geq 0
$$

According to Euler's theory, general solution of differential equations are the same as solutions of ordinary differential equations. We write them in form of linear combination from invariant functions of this kind of derivative:

$$
y(x)=(1+\lambda)^{x} \quad ; \quad x \in Z \quad ; \quad \lambda \in R
$$

It is easy to show that by n-time derivative from proposed invariant function, we have:

$$
y^{(n)}(x)=\lambda^{n}(\lambda+1)^{x} \quad n \in N, x \in Z, \lambda \in R
$$

Now by Substituting above derivatives in the discrete differential equation (1), we obtain:

$$
(\lambda+1)^{x} F(\lambda)=0
$$

Which $F(\lambda)$ is a characteristic Algebraic equation corresponded to (1) differential equation and is written as follows;

$$
F(\lambda)=a_{n} \lambda^{n}+a_{n-1} \lambda^{n-1}+\ldots+a_{1} \lambda+a_{0}
$$


According to nonzero exponential term $(\lambda+1)^{x}$, we will find roots of $F(\lambda)$ and write general solution of (1) differential equation, in the form of following linear combination:

$$
y(x)=c_{1}\left(1+\lambda_{1}\right)^{x}+c_{2}\left(1+\lambda_{2}\right)^{x}+\ldots+c_{n}\left(1+\lambda_{n}\right)^{x} ; \quad \lambda_{i} \neq \lambda j
$$

Now consider equation (1) with the following initial conditions:

$$
y(0)=y_{0}, y^{\prime}(0)=y_{1}, \ldots, y^{(n-1)}(0)=y_{n-1}
$$

We'll reach to follow Algebraic system to evaluate arbitrary constants $c_{1}, \ldots, c_{n}$,

$$
\sum_{k=1}^{n} c_{k} \cdot \lambda_{k}^{j}=y_{j} \quad ; j=0,1, \ldots, n-1
$$

That above system determinant is the same as Vandermonde determinant. Since all $\lambda_{i}, i=1,2, \ldots, n$ are distinct, the determinant is nonzero.

\section{$3 \quad$ Nonlinear difference equations}

To solve this kind of equations, we first remark discrete multiplicative derivative concept. We have discrete multiplicative derivative of discrete function of $f: Z \rightarrow R$ in form of:

$$
f^{[1]}(x)=\frac{f(x+1)}{f(x)} \quad x \in Z
$$

We can easily see that invariant function of this kind of differentiation of discrete function is the following:

$$
f(x)=C^{2^{x}} ; C=\text { Constant }
$$

Where $C$ is an arbitrary constant. [6]

Now, we use the invariant function of this kind of differentiation and consider the general solution of differential equation with discrete multiplicative derivative as:

$$
y(x)=c^{(\lambda+1) x}
$$

Where $\lambda$ is a real number.

It can be shown that,

$$
y^{[1]}(x)=\left(c^{(\lambda+1)^{x}}\right)^{[1]}=\left(c^{(\lambda+1)^{x}}\right)^{\lambda}
$$

The general form of the second order discrete differential equation with multiplicative derivative is:

$$
y^{[11]}(x)=\left(y^{[1]}(x)\right)^{a}((y(x)))^{b} \quad a, b \in R
$$


That initial conditions are:

$$
y\left(x_{0}\right)=y_{0}, \quad y^{[1]}\left(x_{0}\right)=y_{1}
$$

Let's now turn to solve them by using invariant function (10) and substituting in differential equation (11), characteristic equation is obtained:

$$
\lambda^{2}-a \lambda-b=0
$$

Then we obtain roots $\lambda_{1}$ and $\lambda_{2}$, of this equation and by using (10), we write general solution.

Remark 1: Differential equation with discrete multiplicative derivative (11) can be shown as:

$$
y(x+2)=y^{a+2}(x+1)[y(x)]^{b-a-1}
$$

This equation can be written as the following difference equation:

$$
y_{n+2}=y_{n+1}^{a+2} \cdot y_{n}^{b-a-1} \quad a, b \in R
$$

Obviously, it is a nonlinear difference equation.

Example 1: Consider nonlinear difference equation:

$$
y_{n+2}=y_{n+1}^{2}
$$

That is given by initial condition:

$$
y(0)=y_{0}, \quad y^{[1]}(0)=y_{1}
$$

By choosing $a=0, b=1$, above difference equation obtains from (12).

Since characteristic equation of nonlinear equation is $\lambda^{2}-1=0$, its distinct roots are $\lambda_{1}=1, \lambda_{2}=-1$ thus, its general solution is

$$
y(x)=c_{1}^{\left(2^{x}\right)} \cdot c_{2}^{\left(0^{x}\right)}
$$

Where $c_{2}^{\left(0^{x}\right)}=1$, therefore we have:

$$
y(x)=C^{2^{x}}, C=\text { arbitraryconstant }
$$

Now by considering the initial conditions:

$$
y(0)=y_{0} \quad, \quad y^{[1]}(0)=y_{1}
$$

We have the exact analytic solution of this problem in the following form:

$$
y(n)=y_{0}^{2^{n}}, y_{0}=y_{1}
$$


We can easily show that given solution satisfies in initial conditions of problem and nonlinear difference equation.

For example the third order equation is written as follows;

$$
y^{[111]}(x)=\left[y^{[11]}(x)\right]^{a}\left[y^{[1]}(x)\right]^{b}[y(x)]^{c} \quad ; \quad x \in Z
$$

Where $a, b, c$ are real constants.

Therefore, we can write general solution by finding characteristic equation and its roots, as it follows:

$$
\lambda^{3}-a \lambda^{2}-b \lambda-c=0
$$

Hence the general solution of the third equation in the following form is:

$$
y(x)=C_{1}^{\left(\lambda_{1}+1\right)^{x}} \cdot C_{2}^{\left(\lambda_{2}+1\right)^{x}} \cdot C_{3}^{\left(\lambda_{3}+1\right)^{x}} ; x \in Z
$$

Where $\lambda_{i} ; i=1,2,3$ are the distinct real roots of algebraic equation.

\section{Non-Homogenous Linear Difference Equa- tion}

As we saw in the first section, for linear difference equations as:

$$
a_{n} y^{(n)}(x)+a_{n-1} y^{(n-1)}(x)+\cdots+a_{1} y^{\prime}(x)+a_{0} y(x)=0
$$

Which the derivative is a discrite additive derivative.

The general solution is in form of following linear combinations:

$$
y(x)=c_{1}\left(1+\lambda_{1}\right)^{x}+c_{2}\left(1+\lambda_{2}\right)^{x}+\cdots+c_{n}\left(1+\lambda_{n}\right)^{x} \quad ; \quad \text { and } \lambda_{i} \neq \lambda_{j}
$$

In this section we are going to consider and solve the following non-homogenous linear difference equations:

$$
l_{y} \equiv \sum_{j=0}^{n} a_{j} y^{(n-j)}(x)=f(x)
$$

Which non-homogenous term $f(x)$ is in form of a polynomial as follows:

$$
f(x)=\sum_{\rho=0}^{n} b_{\rho} x^{m-\rho}
$$

We use power order function that has been introduced in [3] to use indefinite coefficients method which is used in ordinary differential equations:

$$
\stackrel{k}{X}=A_{x}^{k}=\frac{x !}{(x-R) !}=x(x-1)(x-2) \cdots(x-(k-1)) \quad ; \quad x, k \in N
$$


For to above function we consider:

$$
\stackrel{p+q}{x}=x^{p}\left(x^{q}-p\right) \quad ; \quad p, q \in X
$$

and then $\stackrel{k}{X}$ functions for $k=01,2, \cdots, n$ are linear independent.

According to power order function we can write:

$$
\begin{aligned}
\stackrel{2}{x} & =x \cdot x=x[(x-1)+1]=x^{2}+x \\
\stackrel{3}{x} & =x \cdot x \cdot x=x[(x-1)+1][(x-2)+2] \\
& =x(x-1)(x-2)+2(x-1)+(x-2)+2] \\
& =x^{3}+2 x^{2}+x(x-2)+2 x \\
& \left.=x^{3}+2 x^{2}+x[(x-1)-1)\right]+2 x \\
& =x^{3}+3 x^{2}+x
\end{aligned}
$$

therefore

$$
\begin{aligned}
\stackrel{4}{x} & =x^{3} \cdot x\left[x^{3}+3 x^{2}+x\right] x \\
& =x^{4}+6 x^{3}+7 x^{2}+x
\end{aligned}
$$

By continuing this method, the non-homogenous term can be written as follows:

$$
f(x)=\sum_{\rho=0}^{m} b_{\rho} x^{m-\rho}=\sum_{\rho=0}^{m} \beta_{\rho}{ }^{m-\rho}
$$

Now we solve the second order non-homogenous difference equation by using this method.

$$
y^{\prime \prime}(x)+a y^{\prime}(x)+b y(x)=\sum_{\rho=0}^{m} \beta_{\rho}{ }^{m-\rho}
$$

Consider a particular solution corresponding to non-homogenous term as follows:

$$
y_{0}(x)=\sum_{\rho=0}^{m} c_{0}{ }^{m-\rho}
$$

By additive discrete differentiation of (13) we get

$$
\begin{aligned}
y_{0}^{\prime}(x) & =\sum_{\rho=0}^{m-1} c_{\rho}(m-\rho) \stackrel{m-\rho-1}{x} \\
y_{0}^{\prime \prime}(x) & =\sum_{\rho=0}^{m-2} c_{\rho}(m-\rho)(m-\rho-1) \stackrel{m-\rho-2}{x}
\end{aligned}
$$


By replacing offered solution and its derivatives we have:

$\sum_{\rho=0}^{m-2} c_{\rho}(m-\rho)(m-\rho-1) \stackrel{m-\rho-2}{x}+a \sum_{\rho=0}^{m-1} c_{\rho}(m-\rho) \stackrel{m-\rho-1}{x}+n \sum_{\rho=0}^{m} c_{\rho} \stackrel{m-\rho}{x} \equiv \sum_{\rho=0}^{m} \beta_{\rho} \stackrel{m-\rho}{x}$

If we equalize both sides coefficient of power order function, we have:

$$
\begin{aligned}
& b c_{0}=\beta_{0} \\
& b c_{1}+a c_{0} m=\beta_{1} \\
& b c_{2}+a c_{1}(m-1)+c_{0} m(m-1)=\beta_{2} \\
& \vdots \\
& b c_{k}=a c_{k-1}(m-k-1)+c_{k-2}(m-k-2)(m-k-1)=\beta_{k} \\
& \vdots \\
& b c_{m}+a c_{m-1}+c_{m-2} \cdot 2=\beta_{m}
\end{aligned}
$$

By solving algebraic system (14) we have:

$$
\begin{aligned}
c_{0} & =\frac{\beta_{0}}{b} \\
c_{1} & =\frac{\beta_{1}}{b}-\frac{a_{m}}{b} \cdot \frac{\beta_{0}}{b} \\
c_{2} & =\frac{\beta_{2}}{b}-\frac{a(m-1)}{b}\left(\frac{\beta_{1}}{b}-\frac{a_{m}}{b^{2}} \beta_{0}\right)-\frac{m(m-1)}{b} \cdot \frac{\beta_{0}}{b}
\end{aligned}
$$

\section{Example 2:}

$$
y^{\prime \prime}(x)+a y^{\prime}(x)=3 \stackrel{2}{x}+5 x-1
$$

Consider the assumed solution as:

$$
y_{0}(x)=c_{0} \stackrel{3}{x}+c_{1} \stackrel{2}{x}+c_{2} x
$$

By additive discrete differentiation from each side we have:

$$
\begin{aligned}
& y_{0}^{\prime}(x)=3 c_{0} \stackrel{2}{x}+2 c_{1} x+c_{2} \\
& y_{0}^{\prime \prime}(x)=6 c_{0} x+2 c_{1}
\end{aligned}
$$

By replacing in difference equation we get:

$$
6 c_{0} x+2 x_{1}+3 a c_{0} \stackrel{2}{x}+2 a c_{1} x+a c_{2}=3 \stackrel{2}{x}+5 x-1
$$

If we equalize the equation each side coefficient we get:

$$
\begin{aligned}
& 3 a c_{0}=3 \\
& 6 c_{0}+2 a c_{1}=5
\end{aligned}
$$




$$
2 c_{1}+a c_{2}=-1
$$

By calculating it we have:

$$
\begin{aligned}
& c_{0}=\frac{1}{a} \\
& c_{1}=\frac{5}{2 a}-\frac{6}{2 a} \cdot \frac{1}{a}=\frac{5}{2 a}-\frac{3}{a^{2}} \\
& c_{2}=-\frac{1}{a}-\frac{2}{a}\left(\frac{5}{2 a}-\frac{3}{a^{2}}\right)=-\frac{1}{a}-\frac{5}{a^{2}}+\frac{6}{a^{3}}
\end{aligned}
$$

Which can be get by replacing the coefficient in particular solution of nonhomogenous equation.

Let's now turn to a case that non-homogenous term can be in form of an arbitrary function in this case we use changing parameter method in nonhomogenous ordinary differential equations to each particular solution.

We consider the particular solution as follows:

$$
y_{0}(x)=\sum_{k=1}^{n} c_{k}(x) y_{k}(x) \quad ; \quad x, k \in N
$$

By additive discrete differentiation and replacing them in difference equation (8) according to conditions on $c_{k}^{\prime}(x)$ we reach to the following system:

$$
\left\{\begin{array}{l}
\sum_{k=1}^{n} c_{k}^{\prime}(x)\left[y_{k}^{\prime}(x)+y_{k}(x)\right]=0 \\
\sum_{k=1}^{n} c_{k}^{\prime}(x)\left[y_{k}^{\prime \prime}(x)+y_{k}^{\prime}(x)\right]=0 \\
\sum_{k=1}^{n} c_{k}^{\prime}(x)\left[y_{k}^{(m)}(x)+y_{k}^{(m-1)}(x)\right]=0 \quad ; \quad m=3,4, \cdots, n-1 \\
\sum_{k=1}^{n} c_{k}^{\prime}(x)\left[y^{(m)}(x)+y_{k}^{(m-1)}(x)\right]=f(x)
\end{array}\right.
$$

assume that determinate of above system is not zero, that is:

$$
\Delta(x)=\left|\begin{array}{cccc}
y_{1}^{\prime}+y_{1} & y_{2}^{\prime}+y_{2} & \cdots & y_{n}^{\prime}+y_{n} \\
y_{1}^{\prime \prime}+y_{1}^{\prime} & y_{2}^{\prime \prime}+y_{2}^{\prime} & \cdots & y_{n}^{\prime \prime}+y_{n}^{\prime} \\
\vdots & \vdots & & \vdots \\
y_{1}(n)+y_{1}^{(n-1)} & y_{2}^{(n)}+y_{2}^{(n-1)} & \cdots & y_{n}^{(n)}+y_{n}^{(n-1)}
\end{array}\right| \neq 0
$$

Solution of above system is so

$$
c_{k}^{\prime}(x)=\frac{\Delta_{n k}(x)}{\Delta(x)} f(x)
$$

$\Delta_{n k}(x)$ are minor determinants which known column is instead of mentioned coefficient column. 
By using the meaning of discrete derivative and integral for coefficients of this system, we have:

$$
c_{k}(x)=c_{k}\left(x_{0}\right)+\int_{x_{0}}^{x} \frac{\Delta_{n k}(\xi)}{\Delta(\xi)} f(\xi) \quad ; \quad k=1,2, \cdots, n
$$

So the particular solution of non-homogenous difference equation is written as:

$$
y_{0}(x)=\int_{x_{0}}^{x} \sum_{k=1}^{n} y_{k}(x) \frac{\Delta_{n k}(\xi)}{\Delta(\xi)} f(\xi)
$$

where $\int_{\alpha_{0}}^{\alpha}$ is the discrete additive integral, which has been introduced in [3].

Remark 2: The properties of discrete additive derivative and integration have been considered more in [6].

\section{Conclusion}

As it was mentioned in abstract and introduction of this article, linear and nonlinear equations appear in many natural, physical, engineering systems. Specially environment and population phenomena. So we can solve a large number of these equations in form of either linear or nonlinear as it was mentioned in first and second sections.

\section{References:}

[1] Atkinson, F.V "Discrete and continous Boundary value problems" Academic press, Newyork, 1964.

[2] Gelfond. A, D "calculus of finite difference" Nauka, Moscow, 1967.

[3] Aliev. N \& Bagerove, G \& Izadi. F "Discrete additive analysis" Azarbayjan Tarbiat Moallem university press, Tabriz, IRAN, 1994. (In persion)

[4] Jahanshahi. M "Fundamental of teaching and learning of high school's mathematics" Madreseh press, Tehran, IRAN. 1999 (In persian)

[5] Jahanshahi. M \& Aliev. N "Boundary and Initial Value problems with fractional and irrational order derivatives" AMCA, Fourth ISAAC.York university, Canada, Auguest, 2003.

[6] Aliev. N \& Jahanshahi. M \& Khatami. H. R "Innovation of discrete additive and multiplicative analysis" Research Project, Azad Islamic university of karaj, IRAN, 2003. (unpublished) 
[7] Sebastien Fereuczi \& Christian Mauduit "Transcedence of numbers with alow complexity expansion" Journal of number theory. Vol. 68, Num. 2, December 1997.

Received: February 25, 2006 